\title{
The Diamond Model of Social Response within an Agent-Based Approach
}

\author{
P.R. NAIL ${ }^{a}$ AND K. SZNAJD-WERON ${ }^{b, *}$ \\ ${ }^{a}$ Department of Psychology and Counseling, University of Central Arkansas, USA \\ ${ }^{b}$ Department of Theoretical Physics, Wrocław University of Technology, Wrocław, Poland
}

\begin{abstract}
Models of social response concern the identification and delineation of possible responses to social pressure. Most models are based on simple one-dimensional conceptualizations of conformity and its alternatives even though more sophisticated models have been available for a number of years. The diamond model is perhaps the most refined of the two-dimensional formulations. It is particularly useful in building agent-based models of opinion dynamics because it gives clear and explicit operational definitions of basic types of social response. In fact, the diamond model is actually a ready recipe for a microscopic model of opinion dynamics. Moreover, it fits quite well Einstein's simple but no simpler strategy. In this work, we will present the logic of the diamond model as well as its implications for agent-based modeling.
\end{abstract}

DOI: 10.12693/APhysPolA.129.1050

PACS/topics: 87.23.Ge, 05.50.+q

\section{Introduction}

Quoting maestro Einstein "Everything should be made as simple as possible, but no simpler" [1]. This statement appears to be particularly important for modeling various phenomena in complex social systems. Opinion formation is one of the oldest and most studied subjects in the field of sociophysics [2-10], for reviews read [11-14]. Obviously, public opinion, which can be empirically measured for example as a result of political voting, consumer choices (e.g., Coca-Cola or Pepsi?), etc., is created from individual opinions. Therefore, to build the model, which describes the dynamics of the public opinion, one needs to model how individual opinions change. A key issue is how to model this process in agreement with Einstein's "simple but no simpler strategy". It seems a reasonable approach to look at the ideas of social psychologists because they focus on an individual's behavior when subject to influence from others. Such a strategy should guarantee that the model would not be too simple. Yet, following the ideas of psychologists, physicists might fear that the model would be too complicated. This fear can be allayed, however, when physicists learn of the basic simplicity of most models of social response (for a review see [15]).

In this paper we present a model of social response known as the diamond model. It was initially proposed in $1965[16]$ and has been used by social psychologists for over 50 years. We demonstrate herein that the diamond model fits quite well the "simple but no simpler strategy" [16-18]. In particular, the model gives clear, unambiguous operational definitions for the three most basic and recognized types of social response, namely

*corresponding author; e-mail: katarzyna.weron@pwr.edu.pl conformity, anticonformity, and independence. In Sect. 2 we present the diamond model in its original formulation, and then in Sect. 3, under the $q$-voter dynamics [19-26]. Finally, we discuss how psychological theories can help to build an agent-based model (ABM) of opinion dynamics but also vice versa, namely how the agent-based approach can support psychological theories.

The aim of this paper is twofold. First, we present the diamond model in a way accessible for physicists and show a direct correspondence between social responses of the diamond model and transitions between microstates in the $q$-voter model. Heretofore, such parallels have never been made. We submit that the awareness of such close connections can be pivotal for scientists working on statistical models of social influence from different disciplines. Second, we indicate which parts of the diamond model pave the way for agent-based modeling. We hope these connections will motivate others to further studies based on the diamond model within the majority-vote threshold, as well as other models of opinion dynamics $[11,12,14]$.

\section{The diamond model of social response}

The diamond model's original focus was on operationally defining three basic types of social response conformity and two types of non-conformity, namely anticonformity and independence. What is striking for many statistical physicists is that the original diamond model is limited to dichotomous response possibilities (i.e., agree/disagree). This type of approach is also commonly used in microscopic models of opinion dynamics $[2-5,11-14]$ - models that are often inspired by the famous Ising model. It is always an issue whether opinions should be represented by binary variables. However, surprisingly, such an approach is quite reasonable in that a dichotomous response format is one of the most common in empirical social influence experiments [27]. Moreover, it has been shown empirically that even if the 
response is measured on a continuous scale, the distribution of opinions on "important" issues is typically bimodal with the two peaks coming at extreme values [3].

Willis symbolized possible responses to social influence by a string of three signs: the first sign $(+$ or -$)$ represents the target's initial or pre- exposure position, the second sign $(+$ or -$)$ represents the position advocated by the potential influence source, and the third sign $(+$ or -$)$ represents the target's response to influence (i.e., the post-exposure response). Considering all possible combinations of pre and post agreement/disagreement between the target and source, the Willis scheme yields four basic patterns [16-18]:

$$
\begin{aligned}
& \text { c (conformity): }+-- \text { or }-++ \\
& \text { i (independence): }+-+ \text { or }-+- \\
& \text { a (anticonformity): }++- \text { or }--+ \\
& \text { u (uniformity): }+++ \text { or }--- \text {. }
\end{aligned}
$$

At this point, it is very important to note that the above scheme is not yet the complete diamond model, but only possible outcomes from a single influence trial. The diamond model is a multi-trial model, which means that we need at least two trials to determine the type of social response. Let us illustrate why at least two trials are needed. Imagine for a moment that we do not know the rules governing the system, which is in fact the case in real social systems. We can only observe the behavior of individuals. Individuals discuss on some issue, for example, if they should support $(+)$ or oppose $(-)$ the ruling political party. In this example, a target individual, who initially is supportive $(+)$, is exposed to an influence source who is also supportive $(+)$. After discussing the issue, however, the target individual changes her or his opinion to the opposite state $(-)$. Does this situation necessarily indicate anticonformity? Isn't it possible that such a change can occur not because of the source of influence but due to a change in one's own thinking? In line with the diamond model, this ambiguity is why we need at least two trials to recognize the type of response. This is the reason the diamond model is a multiple-trial model - social responses can only be unambiguously defined over multiple social influence trials $[15,17]$.

In the diamond model, the real social responses are combinations of $(\mathrm{c}, \mathrm{i}, \mathrm{au})$ patterns defined by (1) and measured over $T$ trials. Let us denote by $T_{\mathrm{c}}, T_{\mathrm{i}}, T_{\mathrm{a}}$, and $T_{\mathrm{u}}$ the number of c, i, a, and u patterns out of all $T$ trials. According to the diamond model, we can locate every type and degree of response in the two-dimensional diamond model response space that consists of axis $x$ representing net conformity and axis $y$ representing net dependence:

$$
x=\frac{T_{\mathrm{c}}+T_{\mathrm{a}}}{T}, y=\frac{T_{\mathrm{c}}+T_{\mathrm{u}}}{T} .
$$

Pure forms of social response, namely C (conformity), $\mathrm{I}$ (independence), A (anticonformity) and V (variability) are placed in the corners of a diamond-like response space (see Fig. 1):

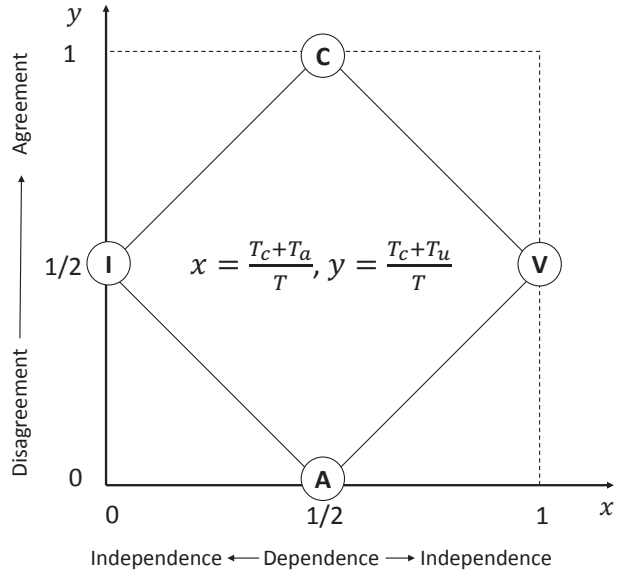

Fig. 1. Diamond model response space [16]. Pure forms of social response, namely $\mathrm{C}$ (conformity), I (independence), A (anticonformity) and V (variability) are placed in the corners of a diamond-like response space.

- Conformity corresponds to $T_{\mathrm{c}}=T / 2, T_{\mathrm{i}}=0$, $T_{\mathrm{a}}=0, T_{\mathrm{u}}=T / 2 \rightarrow(x, y)=(1 / 2,1)$, i.e. is made up of equal parts over social influence trials of the symbolic scheme's conformity $(\mathrm{c}:+--)$ and uniformity $(\mathrm{u}:+++)$. This means that regardless of initial agreement or disagreement, the target's post-exposure response always covaries directly with the position advocated by the influence source.

- Independence corresponds to $T_{\mathrm{c}}=0, T_{\mathrm{i}}=T / 2$, $T_{\mathrm{a}}=0, T_{\mathrm{u}}=T / 2 \rightarrow(x, y)=(0,1 / 2)$, i.e. is made up of the symbolic scheme's independence (i: +-+$)$ and uniformity $(\mathrm{u}:+++)$. This means that the target's response always covaries directly with his or her initial position irrespective of the source's position.

- Anticonformity corresponds to $T_{\mathrm{c}}=0, T_{\mathrm{i}}=T / 2$, $T_{\mathrm{a}}=T / 2, T_{\mathrm{u}}=0 \rightarrow(x, y)=(1 / 2,0)$, i.e. is made up of $(\mathrm{a}:++-)$ and $(\mathrm{i}+-+)$. This means that the target's response always covaries inversely with the source's position.

- Variability (or self-anticonformity) corresponds to $T_{\mathrm{c}}=T / 2, T_{\mathrm{i}}=0, T_{\mathrm{a}}=T / 2, T_{\mathrm{u}}=T / 2 \rightarrow$ $(x, y)=(1,1 / 2)$, i.e. is made up of $(\mathrm{c}:+--)$ and $(\mathrm{a}:++-)$. This means that the target's response always covaries inversely with his or her initial position.

As we see, the diamond model includes the three basic responses, conformity, independence, and anticonformity, but adds a new, fourth possibility, variability, which is actually a second type of independence. Variability is exactly what we have described above in the political example. Here the target shows a change of opinion, but this change is independent of the influence source. Most 
models, not only in social psychology but also in sociophysics, do not provide for such behavior. However, it has been shown empirically that this type of behavior can and does occur. Initially, Willis [16] labeled this fourth response variability. In more recent discussions of the diamond model by Willis and others, however, variability is usually referred to as self-anticonformity $[15,18]$.

\section{The diamond model within the $q$-voter model}

The diamond model is ideal for distinguishing between different types of social response in situations where we do not know the motives, but we see the behavior. When we build an agent-based model, the situation is completely different — we define the rules ourselves. Nevertheless, the diamond model is very helpful because it gives an almost ready recipe for the dynamic rules. The only undefined part of the model is the source of the influence. Is it a single person? If yes, does the influence source represent an authority figure for the target? Maybe the source of influence consists of several people with different initial opinions. If true, how do we define when the source is in the + position? As can be seen, there are many questions but still a lot of freedom in building ABM. Here we focus on the $q$-voter model introduced in [19]. Within this model all individuals are homogeneous, which means that we cannot speak about leaders, authorities etc. The only trait that characterizes an agent is dichotomous opinion $(+/-$, up/down), which is reminiscent of the spin in the Ising model and therefore we have named such a particularly simple agent a spinson (=spin + person) [13]. In the $q$-voter model, a source of the influence consists of $q$ spinsons. What is characteristic of the original $q$-voter model is that the only source of influence is a unanimous group. However, a more general version with the threshold has been introduced in [13].

Within the $q$-voter model it is possible to introduce all types and degrees of social responses described by the diamond model. The question is whether this complexity is really needed. Let us start from the basic qvoter model with only one type of social response, namely conformity. Within such a model, at each time step, a target spinson adopts the opinion of a neighbor if this neighbor belongs to a group of $q$ neighbors all in the same state. A system described by this model eventually always reaches one of two absorbing states - all spinsons "up" or all spinsons "down", i.e. complete consensus, which in reality is quite improbable. Therefore, different kinds of non-conformity have been introduced to the model [20-22]. These changes destroy order (consensus). If we assume that the non-conformity rule is applied with probability $p$, whereas conformity rule with probability $(1-p)$, we can expect an order-disorder (consensusstatus quo) phase transition for a given value of $p$. Can we expect any more or any less? For example, does it matter what kind of non-conformity (anticonformity or independence) we introduce? In other words, would it be possible to recognize, from the level of the society, a world without anticonformity as compared to a world without independence? To answer this question, Nyczka et al. have considered two variants of the $q$-voter model: conformity+independence (model I) and conformity + anticonformity (model A) [20].

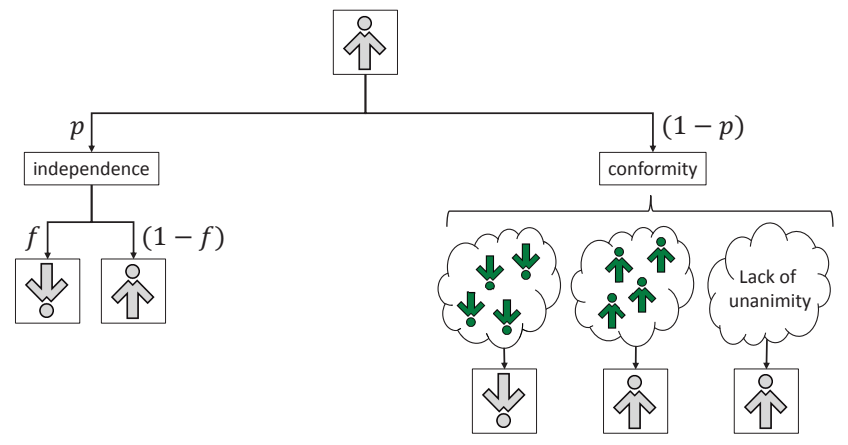

Fig. 2. Model I: an elementary time step of the $q$-voter model with independence. In this example $q=4$ and a target spinson (light gray) has initially opinion +1 .

The algorithm of an elementary step of the q-voter model with independence is the following (see also Fig. 2):

1. Choose randomly one spinson $S_{i}$; go to 2 ;

2. With probability $p$ a spinson is independent, i.e. flips to the opposite position with probability $f$; go to 1 ;

3. With probability $1-p$ a spinson conforms a source of influence, i.e. choose randomly $q$ neighbors of $S_{i}$ (so called $q$-panel) and if all $q$ spinsons are in the same position $S_{i}$ takes the position of the $q$-panel; go to 1.

TABLE I

All possible transitions in the $q$-voter model with independence within the notation proposed by Willis [16]. Patterns (c),(i),(a) and (u) are defined by the Willis scheme (1). Analogously as in the diamond model (C)onformity consists of patterns (c) and (u), (I)ndependence consists of patterns (i) and (u) and (V)ariability consists of patterns (c) and (a).

\begin{tabular}{c|c|c}
\hline \hline \multicolumn{2}{|c|}{$p$} & $1-p$ \\
\hline$f($ V)ariability & $1-f(\mathrm{I})$ ndependece & (C)onformity \\
\hline$-++(\mathrm{c})$ & $-+-(\mathrm{i})$ & $-++(\mathrm{c})$ \\
$--+(\mathrm{a})$ & $---(\mathrm{u})$ & $---(\mathrm{u})$ \\
$++-(\mathrm{a})$ & $+++(\mathrm{u})$ & $+++(\mathrm{u})$ \\
$+--(\mathrm{c})$ & $+-+(\mathrm{i})$ & $+--(\mathrm{c})$
\end{tabular}

To see the analogy between the $q$-voter model with independence and the diamond model, we can now write down explicitly all possible transitions using the same notation as Willis, i.e. as a string of three signs: the first sign $(+$ or -$)$ represents the spinson $S_{i}$ initial position, the second sign $(+$ or -$)$ represents the position of the source of influence i.e. the $q$-panel, and the third sign $(+$ or -$)$ represents position of the spinson $S_{i}$ after an 
elementary time step. As we see from Table I, analogously as in the diamond model, in the $q$-voter model conformity (C) consists of two patterns taken from the Willis scheme, namely $(\mathrm{c})$ and $(\mathrm{u})$. Moreover, we have two types of independent behavior — with probability $f$ patterns (c),(a) appear, which indicate variability (V) and probability $1-f$ we have patterns $(\mathrm{i}),(\mathrm{u})$ i.e. independence (I).

Up until now the $q$-voter model with independence has been analyzed on the complete graph (analytically) $[13,20]$, as well as on various complex networks using Monte Carlo simulations [25, 28]. As usual, to investigate the macroscopic behavior of a system, the magnetization (public opinion) has been measured

$$
m=\frac{1}{N} \sum_{i=1}^{N} S_{i} .
$$

It has occurred that for all investigated networks the critical point $p_{\mathrm{c}}$, below which $m \neq 0$ and above which $m=0$, decreases with $q$ (see Fig. 3). Moreover, a switch from continuous to discontinuous phase transition has been observed. In the case of monoplex networks for $q \leq 5$ the transition is continuous, whereas for $q>5$ discontinuous. For multiplex networks the switch from continuous to discontinuous phase transition has been also observed but for lower values of $q$ [25]. This means that in case of independence we can observe a kind of revolution (rapid jump) between almost fully ordered system (consensus) and disorder (status quo).
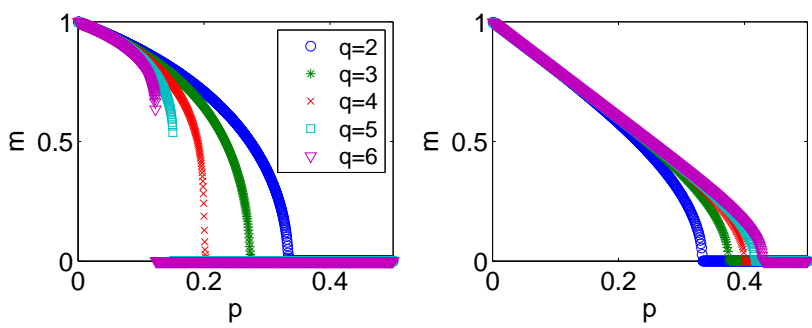

Fig. 3. Magnetization $m$ (public opinion) as a function of non-conformity $p$ for the model on the complete graph. Left part: the $q$-voter with independence and $f=0.5$. For other values of $f>0$ results can be rescaled replacing $p$ by $p f /(1-p+p f)$ [29]. Right part: the $q$-voter with anticonformity.

The algorithm of an elementary step of the $q$-voter model with anticonformity is the following (see also Fig. 4):

1. Choose randomly one spinson $S_{i}$; go to 2 ;

2. Choose randomly $q$ neighbors of $S_{i}$ (so called $q$-panel) and if all $q$ spinsons are in the same position then the $q$-panel will influence the spinson $S_{i}$; go to 3 ;

3. With probability $p$ a spinson is anticonformist, i.e. takes a position opposite to the $q$-panel; go to 1 ;

4. With probability $1-p$ a spinson is conformist, i.e. takes a position of the $q$-panel; go to 1 .

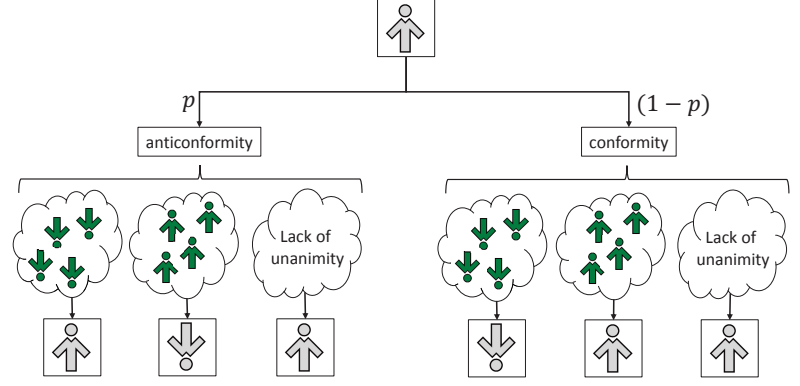

Fig. 4. Model A: an elementary time step of the $q$ voter model with anticonformity. In this example $q=4$ and a target spinson (light gray) has initially opinion +1 .

Again we can write down explicitly all possible transitions using the Willis scheme (see Table II), and again we see a direct correspondence with the diamond model.

TABLE II

All possible transitions in the $q$-voter model with anticonformity within the notation proposed by Willis [16]. Patterns (c),(i),(a) and (u) are defined by the Willis scheme (1). Analogously as in the diamond model (C)onformity consists of patterns (c) and (u), (A)nticonformity consists of patterns (a) and (i).

\begin{tabular}{c|c}
\hline \hline $\mathrm{p}(\mathrm{A})$ nticonformity & 1-p (C)onformity \\
\hline$-+-(\mathrm{i})$ & $-++(\mathrm{c})$ \\
$--+(\mathrm{a})$ & $---(\mathrm{u})$ \\
$++-(\mathrm{a})$ & $+++(\mathrm{u})$ \\
$+-+(\mathrm{i})$ & $+--(\mathrm{c})$
\end{tabular}

In case of anticonformity no switch between continuous and discontinuous phase transition appears, i.e. discontinuous phase transition never appears (see Fig. 3). The second qualitative difference in comparison to model with independence is that the critical point $p_{\mathrm{c}}$ increases with $q$, instead of decreasing. As we see there are significant differences between two versions of the $q$-voter model with non-conformity indicating a need of including two types of non-conformity (independence and anticonformity) in ABM.

\section{Conclusions}

As we have shown, the diamond model of social response is almost a ready recipe to build an agent-based model of opinion dynamics. The only undetermined point is the definition of the source of the influence. In this paper we have defined a source of influence as a unanimous group of size $q$, following the $q$-voter model but other choices are possible and as yet unexplored. Until now most sociophysics models have concentrated on either (a) combining conformity with anticonformity (often named as contrarian behavior $[12,13])$ or (b) combining conformity with pure independence, defined as a lack of movement (stubborn, zealot $[12,13,22]$ ). Other types of 
independent behavior, which are present in the diamond model and correspond to $y=1 / 2$ in the diamond model response space (see Fig. 1), were overlooked. Within the $q$-voter model, this type of behavior corresponds to $f>0$.

In this paper, we have shown a direct correspondence between the different types of social response within diamond and the $q$-voter model. However, one could ask if a simpler model of social response, one that does not distinguish between different types of non-conformity or between different types of independence, might be sufficient. Based on results obtained for the $q$-voter, it appears that the diamond model is the minimal model that fits the simple but no simpler strategy. On the macroscopic scale, results for the model with anticonformity are significantly different than for the model with independence. Surprisingly, independence introduces much more complex, rapid, and unpredictable behavior to the system on the macroscopic level (discontinous phase transition, switch from continuous to discontinuous transition) than does anticonformity. This result, although counterintuitive at first glance, is supported by psychological theories. While it is true that anticonformity and conformity are indeed opposites at the operational level, that is, with respect to the influencee's postinfluence direction of movement, at the conceptual level, the responses are similar in that both indicate behavior that has been influenced by the source [15].

We would like to conclude this paper with the bold assertion that not only can social theories support sociophysics by being a starting point for building ABM, but also vice versa. There are many different models of social response, and one can discuss their advantages and disadvantages at the conceptual level [15]. However, results obtained from ABM show exactly the differences between them, both qualitatively and quantitatively. Although we cannot say conclusively at this point which model is the right one, we can show what kind of behavior would never occur on the level of society without certain types of social response.

\section{Acknowledgments}

This work was partially supported by the National Science Center (NCN, Poland) through grant no. 2013/11/B/HS4/01061.

\section{References}

1A. Calaprice, The Ultimate Quotable Einstein, Princeton University Press, Princeton, New Jersey 2010, p. 384

[2] S. Galam, J. Math. Psychol. 30, 426 (1986).

[3] M. Lewenstein, A. Nowak, B. Latané, Phys. Rev. A 45, 763 (1992).
[4] K. Kacperski, J.A. Holyst, J. Statist. Phys. 84, 169 (1996).

[5] J.A. Hołyst, K. Kacperski, F. Schweitzer, Physica A 285, 199 (2000).

[6] G. Deffuant, D. Neau, F. Amblard, G. Weisbuchr, Adv. Complex Syst. 3, 87 (2000).

[7] P. Sobkowicz, J. Artific. Soc. Soc. Simul. 12, 11 (2009).

[8] K. Kulakowski, Physica A 388, 469 (2009).

[9] P. Sobkowicz, PloS ONE 7, e44489 (2012).

[10] P. Gawronski, M. Nawojczyk, K. Kulakowski, Acta Phys. Pol. A 127, A-45 (2015).

[11] C. Castellano, S. Fortunato, V. Loreto, Rev. Mod. Phys. 81, 591 (2009).

[12] S. Galam, Sociophysics: A Physicist's Modeling of Psycho-political Phenomena, Springer, New Zork 2012.

[13] P. Nyczka, K. Sznajd-Weron, J. Statist. Phys. 151, 174 (2013).

[14] P. Sen, B.K. Chakrabarti, Sociophysics: An Introduction, 1st ed., Oxford University Press, Oxford 2014.

[15] P.R. Nail, S.I. Di Domenico, G. MacDonald, Rev. Gen. Psychol. 17, 1 (2013).

[16] R.H. Willis, Human Relat. 18, 373 (1965).

[17] P.R. Nail, M.D. Van Leeuwen, Personal. Soc. Psychol. Bull. 19, 106 (1993).

[18] P.R. Nail, K. Sznajd-Weron, to be published in: The Psychology of Social Influence: Theory, Techniques and Research, Ed. D. Howard, Nova Science Publishers, New York 2016.

[19] C. Castellano, M.A. Munoz, R. Pastor-Satorras, Phys. Rev. E 80, 041129 (2009).

[20] P. Nyczka, K. Sznajd-Weron, J. Cislo, Phys. Rev. E 86, 011105 (2012).

[21] P. Moretti, S. Liu, C. Castellano, R. Pastor-Satorras, J. Statist. Phys. 151, 113 (2013).

[22] M. Mobilia, Phys. Rev. E 92, 012803 (2015).

[23] M.A. Javarone, T. Squartini, J. Statist. Mech. 10, P10002 (2015).

[24] A.M. Timpanaro, S. Galam, Phys. Rev. E 92, 012807 (2015).

[25] A. Chmiel, K. Sznajd-Weron, Phys. Rev. E 92, $052812(2015)$.

[26] K. Maciejowska, A. Jędrzejewski, A. KowalskaPyzalska, R. Weron, Acta Phys. Pol. A 129, 1045 (2016).

[27] R.W. Robins, R.C. Fraley, R.F. Krueger, Handbook of Research Methods in Personality Psychology.

[28] A. Jędrzejewski, K. Sznajd-Weron, J. Szwabiński, Physica A 446, 110 (2016).

[29] K. Sznajd-Weron, M. Tabiszewski, A. Timpanaro, Euro Phys. Lett. 96, 48002 (2011). 